\title{
Association between myocardial cell apoptosis and calpain-1/caspase-3 expression in rats with hypoxic-ischemic brain damage
}

\author{
HONG ZHAO, MEI XU and GUILAN CHU \\ Department of Pediatrics, Tianjin Medical University General Hospital, Tianjin 300052, P.R. China
}

Received January 7, 2016; Accepted December 29, 2016

DOI: $10.3892 / \mathrm{mmr} .2017 .6341$

\begin{abstract}
The present study aimed to investigate the association between myocardial cell apoptosis and calpain-1/caspase-3 expression in a rat model of hypoxic-ischemic brain damage (HIBD). A total of 64 newborn rats were divided into control $(n=8$; sacrificed on day 7) and HIBD groups $(n=56)$. HIBD group rats were sacrificed 2,12 or $24 \mathrm{~h}$, or $2,3,5$ or 7 days following HIBD ( $n=8 /$ group). A terminal deoxynucleotidyl transferase dUTP nick-end labeling assay was performed to detect myocardial apoptotic cells and calculate the apoptosis index (AI), reverse transcription-polymerase chain reaction was performed to detect myocardial calpain-1/caspase-3 mRNA expression levels and a western blot analysis was conducted to detect calpain-1 protein expression levels. The correlations between calpain-1 and caspase-3 expression levels and AI were analyzed. The results demonstrated that apoptotic myocardial cells in the HIBD groups were markedly increased compared with the control group, with AI peaking in the day 3 group. Caspase- 3 and calpain-1 mRNA expression levels were increased from 2 and $12 \mathrm{~h}$ following HIBD, respectively, with the most elevated levels in the day 2 group. Compared with the control group, calpain-1 protein expression levels were increased from $2 \mathrm{~h}$, with the greatest expression levels in the day 3 group $(\mathrm{P}<0.05)$. Calpain-1 mRNA and protein $(76 / 80 \mathrm{kDa})$ expression levels demonstrated positive linear correlations with $\mathrm{AI}(\mathrm{r}=0.786, \mathrm{P}=0.001$; and $\mathrm{r}=0.853, \mathrm{P}=0.001$, respectively) Caspase $-3 \mathrm{mRNA}$ expression levels were positively correlated with AI $(r=0.894$; $\mathrm{P}=0.001$ ). In conclusion, the present study demonstrated that in rats with HIBD, there is a positive correlation between increased apoptosis of myocardial cells and expression levels of calpain-1 and caspase-3.
\end{abstract}

Correspondence to: Dr Guilan Chu, Department of Pediatrics, Tianjin Medical University General Hospital, 154 Anshan Dao, Heping, Tianjin 300052, P.R. China

E-mail: guilanchu@126.com

Key words: hypoxia-ischemia, myocardial cell, apoptosis, calpain-1, caspase-3

\section{Introduction}

Hypoxic-ischemic brain damage (HIBD) is a primary cause of neonatal death. Asphyxia-induced hypoxia is the pathological basis of damage to various organs. Myocardial tissue is highly aerobic, and the accumulation of acidic metabolites generated by anaerobic glycolysis during asphyxia causes myocardial energy dysmetabolism and adenosine triphosphate (ATP) reduction, resulting in myocardial damage. The greater the degree of asphyxia, the greater the involvement of myocardial cells; thus, the more severe the myocardial damage (1). A previous study demonstrated that, during the process of ischemia-hypoxia, apoptosis and necrosis of myocardial cells occurs, with an increased number of apoptotic compared with necrotic cells in the early stage of ischemia-hypoxia (2).

In hypoxia-ischemia, cells of all tissues exhibit intracellular calcium $\left(\mathrm{Ca}^{2+}\right)$ overload, thus activating numerous $\mathrm{Ca}^{2+}$-dependent proteases. Calpain-1 is a primary protease of the $\mathrm{Ca}^{2+}$-dependent cysteine protease family, and is closely associated with cytoskeleton remodeling, apoptosis and necrosis (1-3). Caspase-3 is an important apoptosis mediator of the caspase family; activated caspase- 3 cleaves and degrades downstream substrates, thus serving a key role in apoptosis. To date, investigations into myocardial cell apoptosis caused by HIBD have been limited. The present study established an HIBD model of 7-day-old neonatal rats, and observed alterations in mRNA and protein expression of calpain-1 and mRNA expression of caspase- 3 in the myocardial cells of HIBD rats. The aim was to analyze the associations between calpain-1, caspase- 3 and apoptosis of myocardial cells in HIBD rats, and to investigate the roles of calpain- 1 and caspase- 3 in the apoptosis of myocardial cells.

\section{Materials and methods}

Animal model. A total of 64 newborn healthy male and female Wistar rats (weight, 12-18 g; age, 7 days) were provided by the Ethics Committee, Laboratory Animal Center, Institute of Radiation Medicine, Chinese Academy of Medical Sciences (Beijing, China). Rats were housed at $21 \pm 2^{\circ} \mathrm{C}$ and a relative humidity of $30-70 \%$ in a 12-h light/dark cycle with free access to food and water. Mean body masses in the experimental groups were not statistically different $(\mathrm{P}>0.05)$. The modified Rice-Vannucci method (4) was used to construct the HIBD 
model in neonatal rats. Rats were randomly divided into control and HIBD groups. The HIBD group was additionally divided into seven subgroups of 8 rats each. Rats were anesthetized with ether (Sigma-Aldrich; Merck Millipore, Darmstadt, Germany), and a median neck incision was performed. In HIBD groups, the left common carotid artery was separated, followed by dual-ligation. The blood vessels were cut between the ligatures. Following surgery, the rats were placed into an anoxic tank supplied with constant $8 \% \mathrm{O}_{2}+92 \% \mathrm{~N}_{2}$ for $2 \mathrm{~h}$ (gas flow $11 / \mathrm{min}$ ). When the rats had recovered, they were returned to the maternal rats for continuous feeding in a consistent environment. A total of eight rats per HIBD group were anaesthetized with $2 \mathrm{ml}$ diethyl ether (Sigma-Aldrich; Merck Millipore) and sacrificed by decapitation at the following time points: 2,12 or $24 \mathrm{~h}$, or 2, 3, 5 or 7 days. The heart was removed quickly and cut into two parts, for storing in liquid nitrogen, or fixing in 10\% neutral formalin (Sigma-Aldrich; Merck Millipore) and embedding in paraffin (Sigma-Aldrich; Merck Millipore) for preparation of sections. In the control group, the common carotid artery was not ligated following separation, and the rats were sacrificed after $2 \mathrm{~h}$.

Detection of myocardial cell apoptosis by terminal deoxynucleotidyl transferase dUTP nick-end labeling (TUNEL) assay. The paraffin sections of heart tissue were dewaxed in xylene (Sigma-Aldrich; Merck Millipore), hydrated with gradient ethanol, and subsequently washed in PBS ( $\mathrm{pH} 7.4$; Fuzhou Maixin Biotech Co., Ltd., Fuzhou, China) for $15 \mathrm{~min}$. Following fixation with blocking solution $\left(3 \% \mathrm{H}_{2} \mathrm{O}_{2}\right.$ dissolved in methanol; Fuzhou Maixin Biotech Co., Ltd.) for $30 \mathrm{~min}$, the sections were rinsed with PBS for $15 \mathrm{~min}$ and soaked in $0.5 \%$ Triton X-100 in PBS (Sangon Biotech Co., Ltd., Shanghai, China) for $5 \mathrm{~min}$, followed by rinsing with PBS for $5 \mathrm{~min}$. TUNEL reaction mixture was added, followed by incubation at $37^{\circ} \mathrm{C}$ for $1 \mathrm{~h}$. Hoescht stain solution $(1: 1,000$; Sigma-Aldrich; Merck Millipore) was added, followed by incubation at room temperature for $10 \mathrm{~min}$. Following three washes with PBS for $5 \mathrm{~min}$, the cells were observed using a BX61 fluorescent microscope (Olympus Corporation, Tokyo, Japan). Apoptosis-positive nuclei appeared green and apoptosis-negative cells appeared blue. The terminal deoxynucleotidyl transferase-free TUNEL mixture served as a negative control. A total of 10 fields of vision were randomly selected from each section. The number of positive cells per 500-1,000 cells was counted under a high magnification, and the percentage of positive cells was calculated as the apoptosis index (AI).

Detection of calpain-1 and caspase-3 mRNA expression levels by reverse transcription-polymerase chain reaction $(R T-P C R)$. Total RNA was extracted from myocardial tissue using TRIzol ${ }^{\circledR}$ (Sangon Biotech Co., Ltd.) according to the manufacturer's protocol, and RT was performed to obtain cDNA for PCR amplification. The RT and PCR kits were purchased from Sangon Biotech Co., Ltd. The RT reaction system was as follows: $4 \mu 1$ PrimeScript Buffer (5X), $1 \mu 1$ PrimeScript RT Enzyme Mix I, $1 \mu 150 \mu \mathrm{mol} / 1$ Oligo Dt Primer, $1 \mu \mathrm{l} 100 \mu \mathrm{mol} / \mathrm{l}$ Random 6 mers, and $13 \mu \mathrm{l}$ total RNA. A Premix Ex Taq PCR system was used. The primers were designed and synthesized by Sangon Biotech Co., Ltd. $\beta$-actin served as an internal control. The primer sequences were as follows: Forward, 5'-GGAGATTACTGCCCTGGCTCCTA and reverse, 5'-GACTCATCGTACTCCTGCTTGCTG for $\beta$-actin (amplified fragment, $150 \mathrm{bp}$ ); forward, 5'-GGGGTG AAGTGGAGTGGAAAG and reverse, 5'-TTAAGGGCGTCA GGTGTAAGG for calpain-1 (amplified fragment, 184 bp); forward, 5'-GAGACAGACAGTGGAACTGACGATG and reverse, 5'-CACGGATCTGTTTCTTTGC for caspase-3 (amplified fragment, $147 \mathrm{bp}$ ). The parameters of the PCR reaction are presented in Table I. Agarose gel electrophoresis (2\%; Sangon Biotech Co., Ltd.) was performed on PCR products, which generated bands with 213, 298 and 749 bp, respectively. GelDoc 2000 gel imaging system and Quantity One software version 4.62 (Bio-Rad Laboratories, Inc., Hercules, CA, USA) were used to detect the band optical density (OD) ratio of the target fragment to $\beta$-actin fragment for semi-quantitative analysis.

Detection of calpain-1 protein expression levels by western blot analysis. The frozen myocardial tissue was ground in liquid nitrogen (Fuzhou Maixin Biotechnology Development Co., Ltd.) and cell lysis buffer (Fuzhou Maixin Biotechnology Development Co., Ltd.) was added to prepare cell lysates. Following centrifugation at $4^{\circ} \mathrm{C}$ at a speed of $256 \mathrm{x} g$ for $10 \mathrm{~min}$, the supernatant was collected for further experiments. The same quantity of protein was collected per group and denatured for $10 \mathrm{~min}$. Proteins $(5 \mu \mathrm{g})$ underwent 10\% SDS-PAGE (Sangon Biotech Co., Ltd.), following which separated proteins were transferred onto polyvinylidene difluoride (PVDF) membranes (Sigma-Aldrich; Merck Millipore) and blocked with 5\% bovine serum albumin solution (Sangon Biotech Co., Ltd.) at $4^{\circ} \mathrm{C}$ overnight. Membranes were incubated with a rabbit anti-rat calpain-1 polyclonal antibody (1:200; catalog no. 3189-100; BioVision, Inc., Milpitas, CA, USA) at $37^{\circ} \mathrm{C}$ for $2 \mathrm{~h}$. Following washing with PBS, the membranes were incubated with a horseradish peroxidase-labeled goat anti-rabbit IgG secondary antibody (1:200; catalog no. 6905-250; BioVision, Inc.) at $37^{\circ} \mathrm{C}$ for 1 h. An Enhanced Chemiluminescence substrate (Fuzhou Maixin Biotechnology Development Co., Ltd.) was added and proteins were imaged using a Gel Imaging system. Proteins with a molecular weight of 76 and $80 \mathrm{kDa}$ were visualized on the PVDF membrane. Quantity One software version 4.1.0 (Bio-Rad Laboratories, Inc.) was used to analyze the OD of protein bands. The OD value of the active segment of calpain was normalized against the value of the total protein to calculate the ratio of the two proteins $(76: 80 \mathrm{kDa})$. The greater the ratio, the greater the degree of positive reaction and target protein activity, whereas a small ratio represented a weaker interaction with the target protein.

Statistical analysis. Data are expressed as the mean \pm standard deviation. SPSS software version 16.0 (SPSS, Inc., Chicago, IL, USA) was used for the analysis. A one-way analysis of variance A one-way analysis of variance followed by Tukey's post hoc test was used for multiple comparisons between groups, and Dixon's Q-test was used for paired comparisons. Pearson's correlation coefficient was performed among different indicators. $\mathrm{P}<0.05$ was considered to indicate a statistically significant difference. 
Table I. Polymerase chain reaction conditions.

\begin{tabular}{lccr}
\hline Thermocycling step & Calpain-1 & Caspase-3 & $\beta$-actin \\
\hline Pre-denaturation & $94^{\circ} \mathrm{C} \times 5 \mathrm{~min}$ & $94^{\circ} \mathrm{C} \times 12 \mathrm{~min}$ & $94^{\circ} \mathrm{C} \times 5 \mathrm{~min}$ \\
Denaturation & $94^{\circ} \mathrm{C} \times 30 \mathrm{sec}$ & $94^{\circ} \mathrm{C} \times 30 \mathrm{sec}$ & $94^{\circ} \mathrm{C} \times 30 \mathrm{sec}$ \\
Annealing & $54^{\circ} \mathrm{C} \times 30 \mathrm{sec}$ & $54^{\circ} \mathrm{C} \times 30 \mathrm{sec}$ & $54^{\circ} \mathrm{C} \times 30 \mathrm{sec}$ \\
Extension & $72^{\circ} \mathrm{C} \times 30 \mathrm{sec}$ & $72^{\circ} \mathrm{C} \times 30 \mathrm{sec}$ & $72^{\circ} \mathrm{C} \times 30 \mathrm{sec}$ \\
No. of cycles & 35 & 75 & 27 \\
Re-extension & $72^{\circ} \mathrm{C} \times 5$ min & $72^{\circ} \mathrm{C} \times 8$ min & $72^{\circ} \mathrm{C} \times 8 \mathrm{~min}$ \\
\hline
\end{tabular}

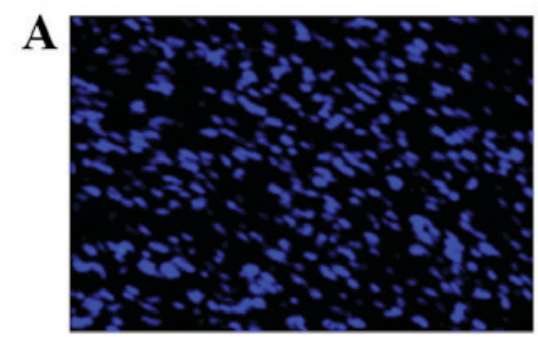

Control group

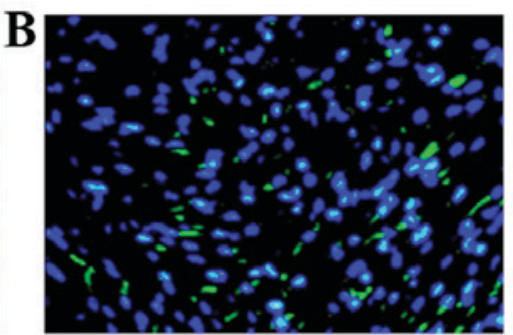

3 Day HIBD group

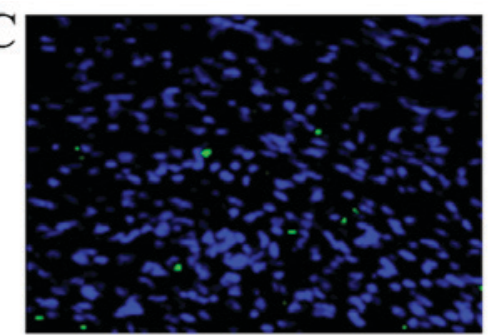

5 Day HIBD group

Figure 1. Myocardial cell apoptosis. Terminal deoxynucleotidyl transferase dUTP nick-end labeling assay demonstrating apoptosis-positive (green) and -negative (blue) cells in the (A) control, (B) 3 day HIBD and (C) 5 day HIBD groups. Magnification, x400. HIBD, hypoxic-ischemic brain damage.

\section{Results}

Myocardial cell apoptosis. As indicated by green-labeled apoptosis-positive myocardial cells, apoptosis was low in the control group (Fig. 1A), whereas the 3 (Fig. 1B) and 5 day (Fig. 1C) HIBD groups exhibited increased levels, particularly in the 3 day HIBD group. Compared with the control group, AI in HIBD groups was significantly increased $(\mathrm{P}<0.05)$, with the greatest score in the 3 day HIBD group. Compared with the control group, AI in all HIBD groups was significantly increased, between $2.5-(2 \mathrm{~h}$ group) and 19.1- (3 day group) fold (Fig. 2).

Alterations of calpain-1 and caspase-3 mRNA and protein expression levels in myocardial tissue. Compared with the control group, mRNA expression levels of calpain-1 were significantly increased in the 12 and $24 \mathrm{~h}$, and 2, 3 and 5 day HIBD groups $(\mathrm{P}<0.05)$, with the greatest expression levels in the 2 day group. Compared with the control group, the mRNA expression levels of caspase-3 were significantly increased in all HIBD groups $(\mathrm{P}<0.05)$, with the exception of the 7 day group, with the greatest expression levels in the 2 day group. Calpain-1 and caspase- 3 mRNA expression levels were decreased in the 3 and 5 day groups compared with the 2 day group; however, levels remained greater than those of the control group ( $\mathrm{P}<0.05$; Fig. 3). Protein expression levels of calpain-1 $(76 / 80 \mathrm{kDa})$ in the HIBD groups were significantly increased compared with the control group, with the exception of the 7 day group, and peaked at 3 days $(\mathrm{P}<0.05$; Fig. 4$)$.

Correlations between myocardial cell AI and calpain-1 and caspase-3 expression. Pearson's correlation analysis revealed that calpain-1 mRNA (Fig. 5A) and protein (76/80 kDa; Fig. 5B) expression levels had positive linear correlations with

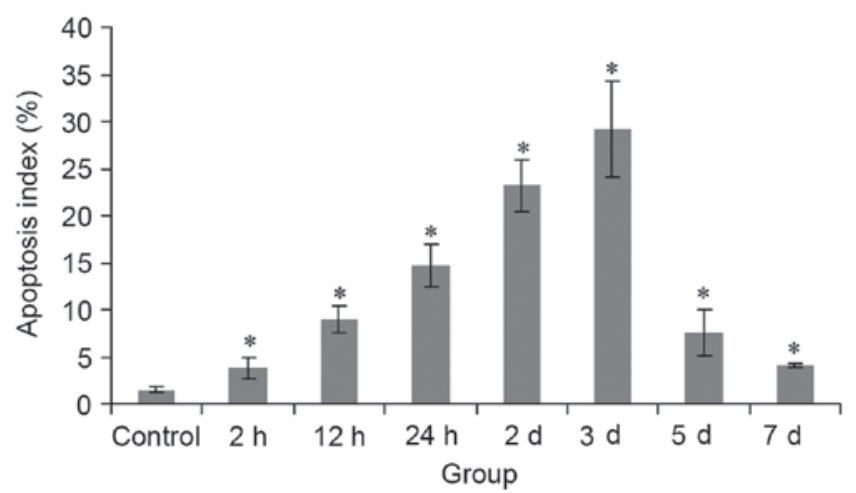

Figure 2. Apoptosis index. Compared with the control group, the apoptosis index in all hypoxic-ischemic brain damage groups was significantly increased, between 2.5- ( $2 \mathrm{~h}$ group) and 19.1- (3 day group) fold. Data are presented as the mean \pm standard deviation. ${ }^{*} \mathrm{P}<0.05$ vs. control group.

$\mathrm{AI}(\mathrm{r}=0.786, \mathrm{P}=0.001$; and $\mathrm{r}=0.853, \mathrm{P}=0.001$, respectively). This indicated that calpain-1 may be involved in hypoxic-ischemic myocardial damage. In addition, the caspase-3 mRNA expression levels were positively correlated with AI $(r=0.894$; $\mathrm{P}=0.001$; Fig. 6), suggesting that the mitochondrial-dependent caspase-3-activated signaling pathway served an important role in hypoxic-ischemic myocardial cell apoptosis.

\section{Discussion}

The calpain protease family is widely distributed in the majority of mammalian tissues, taking the form of zymogens in their resting state, and is primarily activated by $\mathrm{Ca}^{2+}$ in vivo. Once activated, calpains may hydrolyze a large number of intracellular 


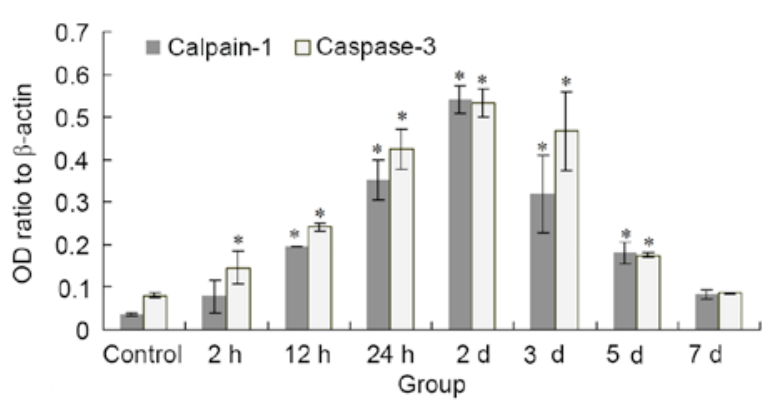

Figure 3. mRNA expression levels of calpain-1 and caspase-3. Semiquantification of polymerase chain reaction analysis. $\beta$-actin served as an internal control. Data are presented as the mean \pm standard deviation. ${ }^{*} \mathrm{P}<0.05$ vs. control group. OD, optical density.

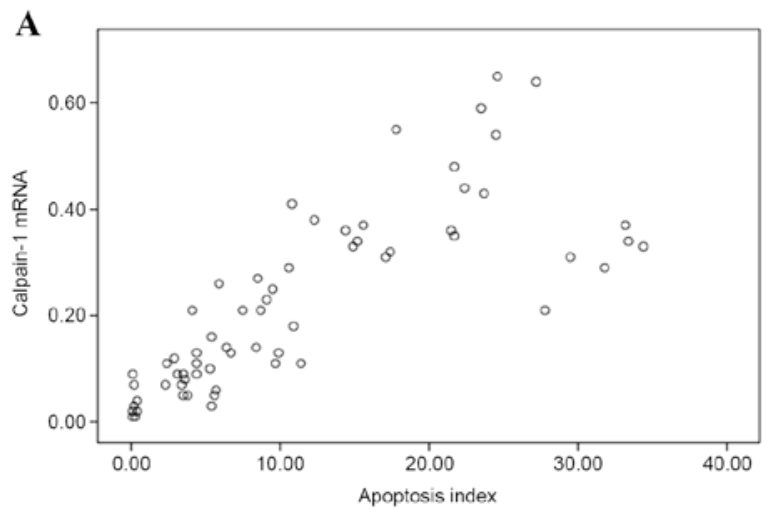

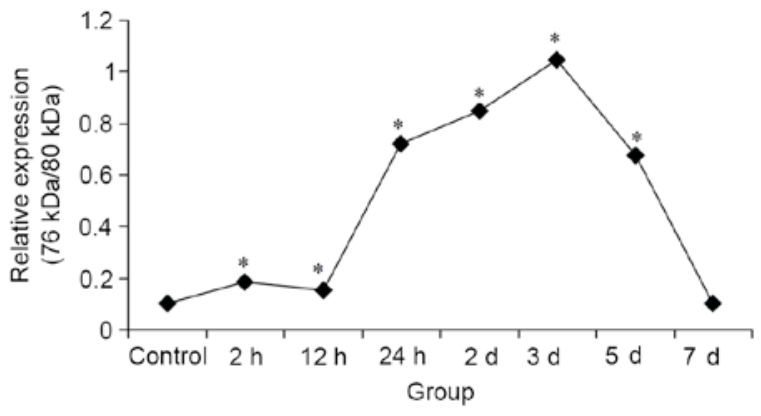

Figure 4. Protein expression levels of calpain-1. Quantification of western blot analysis. Data are presented as the mean \pm standard deviation. " $\mathrm{P}<0.05$ vs. control group.

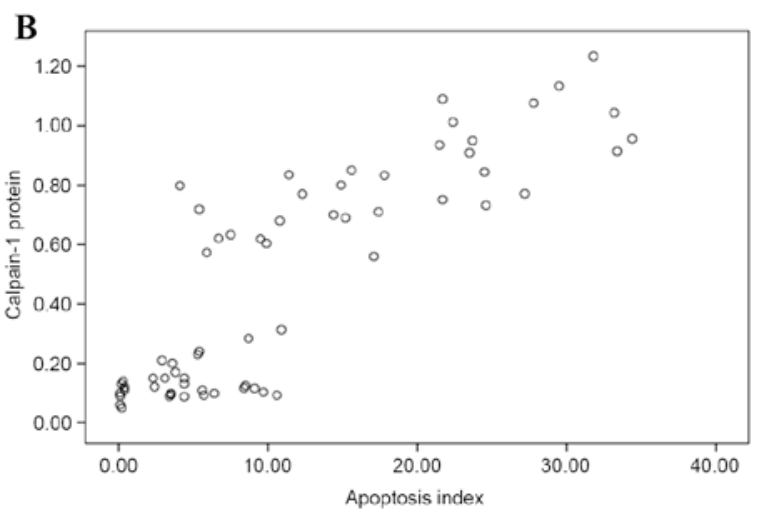

Figure 5. Association of calpain-1 with apoptosis. Correlations of myocardial apoptosis index with calpain-1 (A) mRNA and (B) protein expression levels. $\mathrm{r}=0.786, \mathrm{P}=0.001$; and $\mathrm{r}=0.853, \mathrm{P}=0.001$, respectively.

signaling and structural proteins, involved in pathophysiological processes including cell movement and apoptosis, and cell cycle and gene regulation (1). Calpain-1 primarily exists in the Z-line of sarcomere of myocardial cells, and co-localizes with its endogenous inhibitor calpastatin. Under physiological conditions, intracellular $\mathrm{Ca}^{2+}$ concentrations are maintained at low levels. When stimulated by hypoxia and ischemia, cells instantly increase partial or entire intracellular $\mathrm{Ca}^{2+}$ concentrations via a range of underlying mechanisms, thus overloading intracellular $\mathrm{Ca}^{2+}$. When intracellular $\mathrm{Ca}^{2+}$ concentration is increased, the large subunit of calpain-1 (80 kDa), which has catalytic activity, is hydrolyzed to $76 \mathrm{kDa}$, and the zymogen is activated. A previous study demonstrated that activation of calpain-1 is associated with increasing intracellular free $\mathrm{Ca}^{2+}$ concentrations (5). Excessive activation is involved in the occurrence of ischemic hypoxic cardiac tissue damage $(3,6,7)$. The primary roles of calpain in apoptosis involve regulation of the apoptotic pathways. A previous study on HL-1 cardiomyocytes revealed that when $\mathrm{Ca}^{2+}$ is overloaded, the mitochondrial membrane potential is reduced and calpain activity is upregulated (8). Calpain has been demonstrated to localize inside mitochondria, suggesting that it may cause abnormalities in mitochondrial function (8). During the differentiation of myocardial cells, the death receptor-dependent extrinsic apoptotic signaling pathway is inhibited; therefore, mitochondria serve important roles in the apoptosis of myocardial cells.

Apoptosis serves an important role in maintaining the physiological homeostasis of the body, and growth and development.
Additionally, it is involved in the pathogenesis of numerous diseases. A previous study demonstrated that significant apoptosis of myocardial cells in neonatal rats occurs following HIBD occurrence, and that this abnormal apoptosis remains 7 days later (9). The present study selected 7-day-old Wistar rats to establish an experimental animal model; this avoids shortcomings including single influencing factors in in vitro studies and contamination of cells, and reflects the impact of systemic nerves and body fluids on myocardial tissues following HIBD. The results demonstrated that following HIBD, apoptosis of myocardial cells significantly increased, with the greatest rates at 3 days, and remained significantly increased compared with the control group at 7 days. Furthermore, the present study revealed that following HIBD, the expression levels of myocardial calpain-1 and caspase 3 were significantly increased compared with the control group, and were significantly positively correlated with AI. This suggested that hypoxia and ischemia are associated and may induce apoptosis of myocardial cells and upregulate calpain-1 and caspase-3 expression levels. Therefore, calpain-1 and caspase-3 may co-mediate apoptosis in myocardial cells.

Calpain was previously considered to be closely associated with cell death; however, studies have demonstrated that it may be involved in apoptosis $(10,11)$. Calpain is hypothesized to induce apoptosis in cells under specific stimuli; however, its exact role remains unknown. Calpain-1 may regulate apoptosis of myocardial cells. Ischemia and hypoxia may stimulate the release of intracellular $\mathrm{Ca}^{2+}$ from the endoplasmic reticulum and mitochondria, inducing intracellular $\mathrm{Ca}^{2+}$ overload and triggering a series 


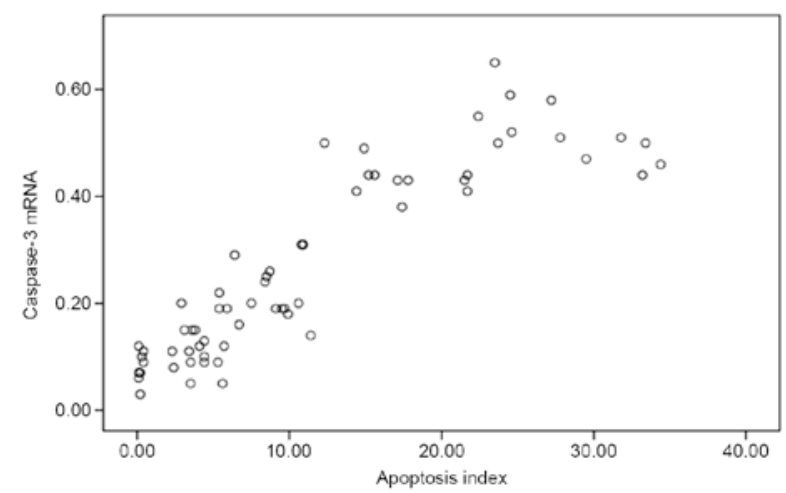

Figure 6. Association of caspase-3 with apoptosis. Correlations of myocardial apoptosis index with caspase- 3 mRNA expression levels. $r=0.894 ; \mathrm{P}=0.001$.

of damaging reactions, known as the 'final common pathway' leading to the death of myocardial cells. Activated calpain has been demonstrated to act on cytoskeletal and membrane proteins, calspectins, and ATP enzymes, altering their structure and function and inducing cell death (12). Caspase-3 is an important protease during the implementation phase of apoptosis. It hydrolyzes calpastatin, the endogenous inhibitor of calpain, and impedes the inhibitory effects of calpastatin towards calpain, thereby increasing calpain activity, forming a positive feedback loop and further promoting apoptosis $(13,14)$. Gafni et al $(15)$ demonstrated that calpain hydrolyzes caspase-7, -10 and -12 , further promoting caspase-induced apoptosis. It is hypothesized that the calpain and caspase families co-mediate apoptosis. Previous studies have demonstrated that calpain may belong to the apoptosis-associated B-cell lymphoma 2 family, and activate additional pro-apoptotic substances including cyclin-dependent kinase 5, apoptotic-protease-activating factor 1, c-Jun N-terminal kinase, transcription factor AP-1 and fos proto-oncogene, AP-1 transcription factor subunit via the hydrolysis signaling pathway, thus mediating apoptosis $(16,17)$. Guo et al (18) revealed that hypoxia induces apoptosis of cardiomyocytes and causes mitochondria to release cytochrome $\mathrm{C}$ into the cytoplasm, upregulating expression levels of upstream caspase- 9 and its downstream effector caspase-3. Therefore, activation of the mitochondria-dependent caspase- 3 signaling pathway serves an important role in hypoxia-induced apoptosis of myocardial cells. In addition, when myocardial cell apoptosis occurs, increased content and activity of calpain-1 inside nuclei further promotes apoptosis (19). Increased activity and expression levels of caspase-3 may directly or indirectly inhibit calpastatin expression levels and activity, thus activating and upregulating calpain-1. Once activated, calpain damages the lysosomal membrane and releases cathepsin, which in turn activates caspase-3, thus forming a pro-apoptotic feedback mechanism. Overactivation of calpain-1 maintains and aggravates pathological conditions; however, it interacts with exogenous and endogenous apoptotic signal transduction pathways, resulting in apoptosis of myocardial cells (20). Calpain-1, calpastatin and caspase-3 are closely associated; however, their specific roles in the apoptosis of myocardial cells require further investigation.

In conclusion, the present study demonstrated that in a rat model of HIBD, myocardial cell apoptosis increased, which positively correlated with increased expression levels of calpain-1 and caspase-3. The role of calpain-1 has received increasing attention; however, its exact underlying molecular mechanisms in the apoptosis of myocardial cells remain to be elucidated. Further investigation is required into apoptosis genes and the underlying mechanisms, using gene transfection and drug studies, to provide novel strategies for the prevention and treatment of HIBD.

\section{References}

1. Goll DE, Thompson VF, Li H, Wei W and Cong J: The calpain system. Physiol Rev 83: 731-801, 2003.

2. Qian W, Xiong X, Fang Z, Lu H and Wang Z: Protective effect of tetramethylpyrazine on myocardial ischemia-reperfusion injury Evid Based Complement Alternat Med 2014: 107501, 2014.

3. Chen X, Zhang X, Kubo H, Harris DM, Mills GD, Moyer J, Berretta R, Potts ST, Marsh JD and Houser SR: Ca2 ${ }^{+}$ influx-induced sarcoplasmic reticulum $\mathrm{Ca}^{+}$overload causes mitochondrial-dependent apoptosis in ventricular myocytes. Circ Res 97: 1009-1017, 2005.

4. Li D, Li X, Wu J, Li J, Zhang L, Xiong T, Tang J, Qu Y and Mu D: Involvement of the $\mathrm{JNK} / \mathrm{FOXO} 3 \mathrm{a} / \mathrm{Bim}$ pathway in neuronal apoptosis after hypoxic-ischemic brain damage in neonatal rats. PLoS One 10: e0132998, 2015.

5. Spencer MJ and Tidball JG: Calpain concentration is elevated although net calcium-dependent proteolysis is suppressed in dystrophin-deficient muscle. Exp Cell Res 203: 107-114, 1992.

6. Zucchi R, Ronca F and Ronca-Testoni S: Modulation of sarcoplamic reticulum function: A new strategy in cardioprotection? Pharmacol Ther 89: 47-65, 2001.

7. Bano D, Munarriz E, Chen HL, Ziviani E, Lippi G, Young KW and Nicotera P: The plasma membrane $\mathrm{Na}^{+} / \mathrm{Ca}^{+}$exchanger is cleaved by distinct protease families in neuronal cell death. Ann N Y Acad Sci 1099: 451-455, 2007.

8. Carpi A, Venerando R, Miotto G, Bertaggia D and Di Lisa F: Calpain and mitochondrial dysfunction in $\mathrm{Ca}^{2+}$ overloaded cardiomyocytes. J Cell Cardiol 42: S105, 2007.

9. Doycheva D, Shih G, Chen H, Applegate R, Zhang JH and Tang J: Granulocyte-colony stimulating factor in combination with stem cell factor confers greater neuroprotection after hypoxic-ischemic brain damage in the neonatal rats than a solitary treatment. Transl Stroke Res 4: 171-178, 2013.

10. Hu H, Li X, Li Y, Wang L, Mehta S, Feng Q, Chen R and Peng T: Calpain-l induces apoptosis in pulmonary microvascular endothelial cells under septic conditions. Microvasc Res 78: 33-39, 2009.

11. Blomgreni K, Leist M and Groc L: Pathological apoptosis in the developing brain. Apoptosis 12: 993-1010, 2007.

12. Sakamoto YR, Nakajima TR, Fukiage CR, Sakai OR, Yoshida YR, Azuma MR and Shearer TR: Involvement of calpain isoforms in ischemia-reperfusion injury in rat retina. Curr Eye Res 21: 571-580, 2000.

13. Wang KK, Posmantur R, Nadimpalli R, Nath R, Mohan P, Nixon RA, Talanian RV, Keegan M, Herzog L and Allen H: Caspase-mediated fragmentation of calpain inhibitor protein calpastatin during apoptosis. Arch Biochem Biophys 356: 187-196, 1998.

14. Kar P, Samanta K, Shaikh S, Chowdhury A, Chakraborti T and Chakraborti S: Mitochondrial calpain system: An overview. Arch Biochem Biophys 495: 1-7, 2010.

15. Gafni J, Cong X, Chen SF, Gibson BW and Ellerby LM: Calpain-1 cleaves and activates caspase-7. J Biol Chem 284: 25441-25449, 2009.

16. Tan Y, Dourdin N, Wu C, De Veyra T, Elce JS and Greer PA: Ubiquitous calpains promote caspase-12 and JNK activation during endoplasmic reticulum stress-induced apoptosis. J Biol Chem 281: 16016-16024, 2006.

17. Lin L, Ye Y and Zakeri Z: p53, Apaf-1, caspase-3, and -9 are dispensable for Cdk5 activation during cell death. Cell Death Differ 13: 141-150, 2006.

18. Guo Z, Liao Z, Huang L, Liu D, Yin D and He M: Kaempferol protects cardiomyocytes against anoxia/reoxygenation injury via mitochondrial pathway mediated by SIRT1. Eur J Pharmacol 761: 245-253, 2015.

19. Zheng D, Wang G, Li S, Fan GC and Peng T: Calpain-1 induces endoplasmic reticulum stress in promoting cardiomyocyte apoptosis following hypoxia/reoxygenation. Biochim Biophys Acta 1852: 882-892, 2015.

20. Smith MA and Schnellmann RG: Calpains, mitochondria, and apoptosis. Cardiovasc Res 96: 32-37, 2012. 